\title{
Nutrición de hierro y lactancia natural en lactantes chilenos
} \author{
Dra. Eva Hertrampf D. ': Sra. Mónica Dinamarca $G_{i}^{2} ;$ T.M. Sandra Llaguno A. ${ }^{1} ;$
Dr. Abraham Stekel $G$.
}

Jrón nutrition in Chilean breast fed infants

\begin{abstract}
Information from rich communities suggests that ison in human milk has a high bioavailability and that breast fed term infants are protected from developing iron deficiency during the first 6 months of life. lron nutrition was evaluated in healthy term infants of low socioeconomic condition living in utban areas (Santiago) receiving breast milk (BF) as the only milk source for 4.6 and 9 months $(68,74$ and 61 subjects respectively), and compared with infants fed iron fortified (FM) and unfortified cow's milk (UM). At 4 months, BF infants showed better iron nutritional status and significant differences ( $\mathrm{p}<0.00 \mathrm{~L}$ ) with both control groups in terms of transferrin saturation $(18.1 \pm 9.9$ vs $11.4+4.3$ and $120 \pm 4.5 \%$ and free ery throcy te protoporphyrin values $(80+29)$ s $90 \pm 29$ and 108 $\pm 33 \mu \mathrm{g} x \mathrm{dl}$. At 9 months of age. $\mathrm{BF}$ and UM groups showed similary deteriorated iron nutrition parame ters, when compared with FM group ( $\mathrm{P}<0.001$ ). At 9 months of age, anemia (hemoglobin $<11 \mathrm{~g} / \mathrm{dl}$ ) was present in $30 \%$ and $29 \%$ ot infants from BM and UM groups respectively, in contrast with only $7 \%$ occurrence in the FM group. These results indicate that chilean breast fed infants from low socioeconomic stratus may develop iron deficiency from 6 months of age on. Dietary supplements of iron shouid be considered for these infants after 4 to 6 months of age.

(Key words: iron, nutrition, human milk).
\end{abstract}

La lactancia natural tiene reconocidas ventajas de tipo nutricional, bioquimico, in munológico, sicológico y económico, las cuales favorecen tanto a la madre como el niño ${ }^{1,2}$ Una de las ventajas cuya importancia ha sido establecida recientemente, es el cfecto sobre la nutrición de hierro durante el primer semestre de la vida. Ha sido descrito que el hietro de la leche humana presenta una alta biodisponibilidad ${ }^{3.4}$ y que lactantes de término no necesitarian recibir suplementos de bierro durante los primeros 6 meses de vida ${ }^{5}$, sin embargo no existe información sistemática en lactantes de áreas menos desarrolladas del mundo, somctidos a condiciones ecolo. gicas distintas. El propésito de este eștudio fue determinar la prevalencia de la deficiencia de hierro. en lactantes de nuestro, pais alimentados con leche materna como la unica fuente lactea por periodos prolongados de tiempo.

\section{MATERIAL Y METODOS}

El estado nutricional de hierro de lactantes que recibieron leche materna como la única fuente láclea fue estudiado a $\operatorname{los} 4,6$ y 9 meses de edad, comparándose

1. Unidad de Hematología, Instituto de Nutrición y Tecnología de los Alimentos, Universidad de Chíe.

2. Profesora de Estado con Mención en Quimica. con lactantes alimentados con leche de vaca fortificada y no fortificada con hierro. Los sujetos para este estudio fueron seleccionados en un corte transversal, a partir de una población de 1500 lactantes beneficiarios del Servicio de Salud, provenientes de áreas urbanas, seguidos desde el nacimiento hasta los 15 meses de edad en 7 consultorios del Servicio de Salud en el Area Central de la Región Metropalitana. En esta población, la lactancia natural era estimulada actíramente. Después del destete espontáneo, la mitad de los niños recibían leche en polvo entera reconstituida al $10 \%$ fortificada con $15 \mathrm{mg}$ de hierro (como sulfato ferroso) y 100 $m g$ de ácido ascótbico $\times 100 \mathrm{~g}$ de polvo (LF); la otra mitad tomaba la misma leche pero no fortificada (LP). La alimentación sólida fue introducida en todos los lactantes desie los 4 meses de vida (vegetales $y$ carne). A los 4, 6 y 9 meses de edad se escogieron 68,74 y 61 lactantes que estuviesen recibiendo leche materna como única fuente láctea (LM) y niños de las mismas edades, que liabian abandonado la lactancia natural en forma espontánea ante de los 2 meses de vida. entre los que estaban recibiendo la leche fortificadil (LF) $(N=$ $52 ; 48$ y 55 ) y la leche no fortificada (LP) $\mathrm{N}=$ 54,60 y 78). Todos los lactantes seleccionados habían nacido de término, sanos, con pesos de nacimiento mayores que $2.500 \mathrm{~g}$ y se encontraban, en el momento del estudio, sobre el percentjl $10 \mathrm{de}$ las tablas del Centro Nacional de Éstadísticas de

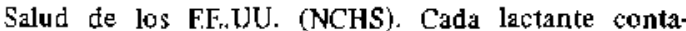
ba con un registro mensual del tipo de leche $y$ de los alimentos sólidos que recibía, según información obtenida en domicilio por una enfermera universitaria. Los tres grupos en las diferentes edades resultaron homogéneos en peso de nacimiento, crecimien- 
to, morbilidad y nivel socioeconomico. La cantidad de hiero proveniente de los alimentos sólidos resultó despreciable en todos los grupos. La nutrición de hierro fue evaluada desde muestras de sangre venosa, mediante determinaciones de concentración de hemoglobina (Hb), hierro sérico, capacidad de unión a la transferrina $(\mathrm{Fe} / \mathrm{IBC})^{6}$, protoporfirina critrocitaria libre $\left(\mathrm{Fl} \mathrm{P}^{7}\right.$ y ferritina sérica $(\mathrm{FS})^{8}$. E] protocolo fue aprobado por el Comité de Etica pa. ra investigación en humanos del lnstituto de Nutrición, obteniéndose también el consentimiento verbal de los padres antes de la inclusión de los sujetos en el estudio. En el análisis estadístico se emplearon los promedios aritméticos, geométricos, desviación típica y prueba t de Student.

\section{RESULTADOS}

A los 4 meses de edad, las concentraciones de hemoglobina fueron similares en los 3 grupos. Los lactantes alimentados al pecho tenían mayores saturaciones de transferrina $(p<0,001)$ y valores de protoporfirina eritrocitaria libre signi. ficativamente menores que los grupos controles ( $\mathrm{p}<0,001)$. En los grupos de LM y LF los valores promedios de ferritina sérica fueron significativamente más al tos que en el grupo LP ( $p$ $<0,001$ ) (Tabla 1).

En los lactantes de 6 meses de edad, Jos valores promedios de los parámetros hematológicos en el grupo LM fueron similares a los del grupo LP: a esta edad en ambos grupos el estado de nutrición de hierro estaba más deteriorado que en grupo LF (Tabla 2).

A los 9 meses de edad, el deterioro de Jos parámetros, exceptuando la FS, se acentuó en los grupos LM y LP, siendo el promedio de FS similar entre los grupos LM y LF y significativamente superior en ambos $(p<0,001)$ que en el grupo LP (Tabla 3).

Al definir como deficientes en hierro a lactan- tes con 2 o más parámetros de laboratorio ánormales, a los 4 meses no se encontraron signos de deficiencia en los grupos LM y LF. A los 6 meses de edad, el grupo LM no se distinguía del grupo LP en el porcentaje de sujetos con signos de deficiencia de hierro; mientras que a los 9 meses la mitad de los sujetos tenían signos de deficiencia del mineral en los grupos LM y LP (figura 1).

\section{DISCUSION}

La de hierro es la deficiencia nutricional más prevalente hoy en dia, en países ricos y pobres. Encuestas realizadas en Chile entre lactantes de nivel socioeconómico bajo ${ }^{9}$ han demostrado anemia en $33 \%$ de los lactantes entre 6 y 24 meses de edad y en $46 \%$ signos de deficiencia de hierro. Uno de los factores que puede contribuir a la alta prevalencia de esta deficiencia en lactantes meno. res de 1 año es la corta duración de la tactancia materna. En. las últimas décadas ha existido una tendencia mundial a la disminución de la lactan. cia natural de la cual Chile no ha escapado. Encuestas recientes realizadas en Santiago indican que sólo $40 \%$ de los lactantes están recibiendo Jeche materna a los 3 meses de edad y $25 \%$ a los 4 meses $^{10}$.

Desce hace mucho tiempo se han descrito diferencias en la nutrición de hierro entre lactantes alimentados al pecho $y$ artificialmente, sin embargo el tema aún es motivo de debate: Mackay y cols en $1928^{11}$ y Horan y cols en $1950^{12}$ encontraron concentraciones de hemoglobina más altas en lactantes alimentados at pecho que en los alimentados en forma artificial. Ningún otro parâmetro đe nutrición de hierro fue medido en estos estudios. En 1954, Feuillen ${ }^{13}$

Tabla 1

Nutrición de hierro en lactantes a los

4 meses de edad.

\begin{tabular}{|c|c|c|c|c|c|}
\hline & $\mathrm{N}$ & $\begin{array}{r}\mathrm{Hb}^{*} \\
(\mathrm{gxdl})\end{array}$ & $\begin{array}{c}\text { Fe } x \text { IBC } \\
(\%)\end{array}$ & $\begin{array}{c}\text { FEP } \\
(\text { ugxdl) }\end{array}$ & $\begin{array}{c}\text { SF\# } \\
\left(u_{g} \times L\right)\end{array}$ \\
\hline $\begin{array}{l}\text { Leche (LP) } \\
\text { no fortificada }\end{array}$ & 54 & $11,84 \pm 0,90$ & $11,4 \pm 4,3$ & $90 \pm 29$ & $\begin{array}{c}25,0 \\
(14,9-42,5)\end{array}$ \\
\hline $\begin{array}{l}\text { Leche (LM) } \\
\text { humana }\end{array}$ & 68 & $11,80 \pm 0,80$ & $18,1 \pm 9,9 \mathrm{a}$ & $80 \pm 29 \mathrm{~b}$ & $\begin{array}{c}46,9 c \\
(22,9-96,1\end{array}$ \\
\hline $\begin{array}{l}\text { Leche (LF) } \\
\text { fortificada }\end{array}$ & 52 & $11,75 \pm 0,90$ & $12,0 \pm 4,5$ & $108 \pm 33$ & $\begin{array}{c}49,1 \\
(28,8-83,7)\end{array}$ \\
\hline
\end{tabular}

* Promedio y $1 \mathrm{DE}$, \# promedio geométrico y rango de $1 \mathrm{DE}$.

El grupo LM difiere significativamente de los gropos LP y LF ap $<0,001$; de LF bp $<0,001$ y de LP cp $<0,001$. 
Tabla 2

Nutrición de hierro en lactantes a los 6 meses de edad.

\begin{tabular}{|c|c|c|c|c|c|}
\hline - & $N$ & $\begin{array}{l}\text { Hb* } \\
(\mathrm{g} \times d \mathrm{~d})\end{array}$ & $\begin{array}{c}F e x J B C \\
{[\%]}\end{array}$ & $\begin{array}{c}\text { FEP } \\
\text { (ugxdl) }\end{array}$ & $\begin{array}{c}\text { SF \# } \\
\text { (ugxL) }\end{array}$ \\
\hline $\begin{array}{l}\text { Leche (LP) } \\
\text { no fortificada }\end{array}$ & 60 & $11,63 \pm 1,13$ & $15,0 \pm 7,4$ & $79 \pm 29$ & $\begin{array}{c}23,6 \\
(10,2 \cdot 54,6)\end{array}$ \\
\hline $\begin{array}{l}\text { Leche (LM) } \\
\text { humana }\end{array}$ & 74 & $11,62 \pm 0,78$ & $17,3 \pm 8,8$ & $82 \pm 26$ & $\begin{array}{c}20,1 \\
(8,4-47,1)\end{array}$ \\
\hline $\begin{array}{l}\text { Leche (LF) } \\
\text { fortificada }\end{array}$ & 48 & $12,12 \pm 0,93$ & $12,1 \pm 4,2 b$ & $93 \pm 29 \mathrm{c}$ & $\begin{array}{c}33.7 d \\
(18,4-61,6)\end{array}$ \\
\hline
\end{tabular}

* Promedio y 1 DE, \#Pronedio Geométrico y rango de $1 \mathrm{DE}$.

Los grupos L.Yy LP difieren significatiuamente de LF: ap $<0,005)$; bp $<0,00$ i ; cp $<0,05 ;$ dp. $<0,001$.

Tabla 3

Nutrición de hierfo a los 9 meses de edad

\begin{tabular}{lccccc}
\hline & $\mathrm{N}$ & $\begin{array}{c}\mathrm{Hb} \\
(\mathrm{gxdl})\end{array}$ & $\begin{array}{c}\text { FExiBC } \\
(\%)\end{array}$ & $\begin{array}{c}\text { FEP } \\
\text { (ugxd) }\end{array}$ & $\begin{array}{c}\text { SF } \\
\text { (ug:L) }\end{array}$ \\
\hline $\begin{array}{l}\text { Leche (LP) } \\
\text { no fortificada }\end{array}$ & 78 & $11,43 \pm 1,22$ & $9,3 \pm 5,4$ & $99 \pm 53$ & $\begin{array}{c}9,8 \\
(4,7-20,4)\end{array}$ \\
\hline $\begin{array}{l}\text { Leche (LM) } \\
\text { humana }\end{array}$ & 61 & $11,35 \pm 0,99 \mathrm{a}$ & $9,5 \pm 3.8 \mathrm{~b}$ & $98 \pm 34 \mathrm{c}$ & $\begin{array}{c}17.0 \mathrm{~d} \\
(7,1-41,0)\end{array}$ \\
\hline $\begin{array}{l}\text { Leche (LF) } \\
\text { fortificada }\end{array}$ & 55 & $12,59 \pm 0.89$ & $14,4 \pm 6,0$ & $66 \pm 21$ & $\begin{array}{c}20.3 \\
(11,1-39,2)\end{array}$ \\
\hline \hline
\end{tabular}

* Promedio y l DE, \#Pronedio gconétrico y rango de $1 \mathrm{DE}$.

Los grupos LM y LP difieren significativamente de LF: ap, bp y cp<0,001 Grupos LM y LF difieren de LP, ap, bp, ep y dp $<0,001$.

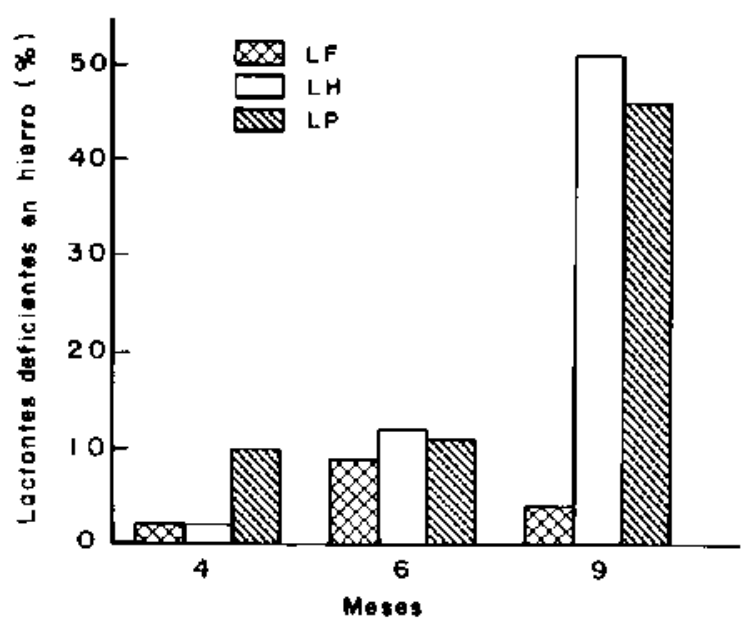

Figura 1. Porcentaje de lactantes con dos o más criterios de defíciencia de hierro. Límites inferiores empleados $\mathrm{Hb}<10 \mathrm{~g} \times$ dl a $\operatorname{los} 4$ y 6 meses de edad, $<11 \mathrm{~g} \mathrm{x}$ dl a los 9 meses. $\mathrm{Fe} / \mathrm{IBC}>9 \%$ ГEP $120 \mathrm{ug} / \mathrm{dl}$ o $\mathrm{SF}<10$ $\mathrm{ug} / \mathbf{L}$ en cada grupo. explicó este hecho por la alta proporción de hierro que es absorbida de la Jeche humana empleando una técnica de balance en sólo dos sujetos. Posteriormente. McMillan y Saarinen con una técnica basada en el uso de radioisótopos mostraron los mismos resuitados 3,4 .

En las últimas dos décadas se han realizado varios estudios para determinar el grado en que se refleja en los lactantes la mejor biodisponibilidad del hierro de la Jeche materna. Sin embargo, todos estos trabajos han sido realizados en comunidades desarrolladas. Woodruff ${ }^{14}$, siguiô longitudinalmente a un pequeño grupo de lactantes alimentados en forma natural, encontrando sólo diferencias en el IBC, al compararlo con un grupo control alimentado con una leche de vaca no fortificada con hierro, la $\mathrm{Hb} y$ el Fe eran similares. Desafortunadamente estos dos grupos no resultaron comparables por la diferencia de ingesta de hierro en sus dietas. En un estudio con 
un mayor número de sujetos, Jos lactantes alimentados con pecho exclusivo no presentaban ningún signo de deficiencia de hierro a los 6 meses de edad, sin embargo a los 9 meses, $4 \%$ tenían deficiencia de hierro. Los lactantes alimentados con leche de vaca presentaban deficiencia de este mineral a los 4 meses de edad; $y$ los que recibieron fórmulas fortificadas con hierro no mostraron signos de deficiencia durante los primeros 12 meses de vida. ${ }^{5}$ Picciano y cols encontraron indices hematológicos similares en lactantes alimentados al pecho $y$ en aquellos que recibieron una fórmula fortificada a los 6 meses de edad 15 . Owen y cols demostraron nuevamente que los Jactantes alimentados al pecho durante los primeros 6 meses de vida aun cuando no hayan recibido aporte de hierro fuera del intrínseco de la leche materna, no sufrian deficiencias de hierro a los 6 meses de edad $\mathbf{1 6}$.

Este estudio confirma que los lactantes alimentados con pecho tienen mejor nutrición de hierto que los alimentados con leche de vaca no fortificada durante los primeros meses de vida. Durante la segunda mitad del primer año, sin embargo, lactantes alimentados con pecho, que recibían alimentos sólidos, desarrollaron deficiencias de hierro tan severas como los lactantes alimentados con leche no fortificada. Comparados con los lactantes europeos del estudio de Saarinen, la deficiencia de hierro en lactantes amamantados chilenos se desarrolló más precoemente. No contamos con una explicación para este hallazgo en base a la información disponible. Sugerimos la necesidad de realizar estudios de seguimiento con el fin de controlar mas estrictamente variables intervinientes tales como evolución pondoestatural, composición de la dieta, morbilidad, etc.

Sin embargo, estos resultados sustentan la necesidad de suplementar con hierro, desde los 4 meses de vida, tanto a los lactantes destetados como a los que reciben una lactancia natural prolongada.

\section{RESUMEN}

Información proveniente de comunidades desarrolladas sugiere que el hierro en la leche humana es de alta biodisponibilidad y que los lactantes de término alimentados con el pecho están protegidos de la deficiencia de hiecro durante los primeros 6 meses de vida. Se estudió el estado de nutrición de hierro de lactantes de término, sanos de 4,6 y 9 meses de edad ( $n=68$, 74 y 61 sujetos respectivamente) de bajo nivel socioeconómico, provenientes de áreas urbanas de Santiago, alimentados con leche materna como única fuente láctea (LM), comparándolos con 2 grupos de lactantes destetados antes de los 3 meses de edad, que recibieton, respectivamente, leche fortificada con hierro (LF) y leche no fortificada (LP). A los 4 meses, el grupo LM presentaba mejor nutrición de hierro, mostrando diferencias significativas con ambos grupos controles en la saturación de la transferrina $18,1 \pm 9,9$ vs $11,4 \pm 4,3$ y $12,0 \pm 4,5)$ y en la protoporf irina eritrocitaria libre $(80 \pm 29$ vs $90 \pm 29$ y $108 \pm 33$ ). Los promedios geométricos de ferritina sérica en los grupos LM y LF fueron significativamente mayores $(p<0,001$ ) que en el grupo LP $(46,9$ y 49,1 vs 25,0$)$. A los 6 y 9 meses de edad el grupo LM no difería del grupo LP en ninguno de los parámetros medidos, mostrando ambos valores que indican deterioro del estado nutricional de hierro, al ser comparado con el grupo LF. A la edad de 9 meses, 30 y $29 \%$ de los lactantes de los grupos LM y LP, respectivamente, tenían anemia (hemoglobina $11 \mathrm{~g} / \mathrm{dl}$ ) vs $7 \%$ en el grupo LF. Los resultados sugieren que los lactantes chilenos alimentados con pecho pueden presentar signos de deficiencia de hierro a los 6 meses de vida. La suplementación con hierro debe ser considerada, por ende, en los lactantes alimentados en forma natural después de los 4 o 6 meses de edad.

\section{REFERENCIAS}

1. Jellife, $D B$.: World trends in infant feeding. Am $\mathbf{J}$ Clin Nutr 29: $1227,1976$.

2. Jellife D., Jellife P.: Breast is best. Modern meanings. Lancet 17: $912,1977$.

3. McMilan $J$, Landaw $S$, Oski $F$.: Iron suficiency in breast fed infants and the availability of iron from human milk. Pediatrics 58: 686, 1976

4. Saarinen U., Simes M. Daliman P.: Iron absortion in infants: high bioavailability of breast milk íron as indicated by the extrinsec tag method of iron absorption and by the concentration of serum ferritin. J Pediatr 91: 36, 1977.

5. Sacrinen $U .:$ Need for iron supplementation in infants on prolonged breast feeding. J Pediatr 93: 17?, 1978

6. Fischer D., Price D.: A simple serum jron method using the now sensitive chromogen tripyridyl-strialine, Clin Chem 10: 21, 1964.

7. Heller SR, Labbe RE., Nutter J.A.: A simplified assay for porphytins in whole blood. Clin Chem 17: 525,1971 .

8. Anónimo: Gamma Dab (1251) ferritin radioimmuno-assay-Kit. Ctinjeal Assays Division of Travenol Lab. Inc, Cambridge, Mass, USA.

9. Rios E., Olivares M., Amar $M$, et al.: Evaluation of iron status and prevalence of iron deficiency in infants in Chile. In: Underwood BA, ed. Nutrition Intervention strategies in national development. New York: Academic Press, 273, 1983.

10. Stekel $A$. , Mardones $F_{+}$, Hertrampf $E_{\text {, }}$ Breastfeeding practices and use of supplemental foods. In: Alan R. Liss, ed. Malnutrition: Determinants and consecuences. New York: 139, 1984. 
11. MacKay H.: Anemia in infancy: lts prevalence and prevention. Arch Dis Child 3: 1175, 1928.

12. Horan M.: Studies in anaemia of infancy and childhood. The hamoglobin, red cell count and packed cell volume of normal english infants during the first year of life. Arch Dis Child 25: 110, 1950.

13. Feuillen $Y_{*}$, Plumier $M$. .: Iron metabolism in infants. I. The intake of iron in breast feeding and artificial feeding (milk and milk foods). Acta Paediat 41 : 138,1952 .
14. Woodruff C., Latham C., McDavid S.: Iron nutrition in the breast fed infant. J Pediatr 90: 36, 1977.

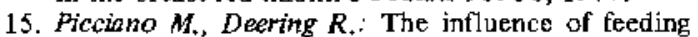
regimes on iron status during infancy. Am J Clin Nutr 33: 746, 1980.

16. Owen $G$., Gary $E$., Hooper $E$ et al.: Iron nutriture of infants exclusively breast fed in the first frve months. Pediatr Res 99: 237, 1981. 